\title{
Crizotinib - Molekulare Therapie des Lungenkarzinoms
}

\section{Crizotinib - Molecular Therapy for Lung Cancer}

Autoren

Institute
A. Gröschel ${ }^{1}$, A. Warth ${ }^{2}$, N. Reinmuth ${ }^{3}$

${ }^{1}$ Ambulantes Aachener Zentrum für Lungenheilkunde, Luisenhospital, Aachen

${ }^{2}$ Institut für Pathologie, Universitätsklinikum Heidelberg

${ }^{3}$ Lungenclinic Grosshansdorf
Bibliografie

DOI http://dx.doi.org/

10.1055/s-0032-1326365

Pneumologie 2013; 67: 205-208

(c) Georg Thieme Verlag KG

Stuttgart · New York

ISSN 0934-8387

Korrespondenzadresse

Dr. med. Andreas Gröschel

Ambulantes Aachener Zentrum

für Lungenheilkunde

Luisenhospital

Boxgraben 95

52064 Aachen

Dr.A.Groeschel@t-online.de

Serienherausgeber

C. Grohé, Berlin

N. Reinmuth, Grosshansdorf

\section{Zusammenfassung}

$\nabla$

Die anaplastische Lymphomkinase (ALK) kann durch eine Gen-Translokation überaktiviert werden und dadurch ein Schlüsselonkogen bei der Progression von Tumorzellen werden. Bei ungefähr $5 \%$ aller Patienten mit fortgeschrittenem nicht-kleinzelligem Lungenkarzinom (NSCLC) lässt sich mittels FISH (Fluoreszenz-in-situ-Hybridisierung) ein Fusionsgen, bestehend aus EML4 (echinoderm microtubule-associated protein-like 4) und ALK, nachweisen, welches zu einer Überaktivierung von ALK führt. Weitere Labortechniken können eine veränderte ALK-Expression nachweisen, wobei die Vergleichbarkeit mit FISH-Ergebnissen unterschiedlich ist. Crizotinib ist eine kleinmolekulare Substanz, die die ALK-Tyrosinkinaseaktivität hemmt. Die Behandlung mit Crizotinib von vorbehandelten NSCLC-Patienten, bei denen EML4-ALK-Fusionsgene nachgewiesen wurden, zeigte in klinischen Studien ein verbessertes Ansprechen und verlängertes progressionsfreies Überleben (PFS) im Vergleich zur Standard-Chemotherapie. Im Folgenden sollen Nachweismethoden und klinische Daten dargestellt werden.

\section{Einleitung}

\section{$\nabla$}

Bei der Therapie des Lungenkarzinoms gewinnen molekulare Veränderungen im Tumorgenom zunehmend an Bedeutung. Neben der Mutation im EGF-Rezeptor wurde 2007 zuerst über eine erworbene EML4- und ALK-Translokation berichtet, die zur Produktion eines ALK-Onkoproteins bei Patienten mit nicht-kleinzelligem Lungenkarzinom führte. Über Phosphatidylinositol 3-kinase (PI3K) und HSP 90 kommt es dann zu einer Zellproliferation und Zellüberleben. Crizotinib, ein oraler Tyrosinkinaseinhibitor, hemmt ALK und zeigt ein deutliches Tumoransprechen in Patienten mit dieser Translokation. Die Zulassung er-

\section{Abstract \\ $\nabla$}

The anaplastic lymphoma kinase (ALK) can act as a key oncogenic driver after activation by means of processes such as gene rearrangement. In approximately $5 \%$ of patients with advanced nonsmall cell lung cancer (NSCLC), an oncogenic fusion gene of echinoderm microtubule-associated protein-like 4 (EML4) and ALK has been detected using fluorescence in situ hybridisation (FISH). Moreover, various methods including immunohistochemistry and PCR-based assays can be used for analysing ALK expression. Clinical data have been generated for crizotinib, a small molecule inhibitor of the ALK receptor tyrosine kinase, demonstrating a substantial improvement of objective response rate and prolonged progression-free survival (PFS) compared to standard chemotherapy in pretreated NSCLC patients harbouring EML4-ALK fusion genes. In the current review, recent data on the detection and inhibition of ALK in advanced NSCLC are summarised.

folgt durch die EMA im November 2012 zur Behandlung von Erwachsenen mit vorbehandeltem EML4-ALK-positivem fortgeschrittenem nichtkleinzelligem Lungenkarzinom unter dem Handelsnamen Xalkori. Dies ist somit nach der EGFRMutation eine weitere Gruppe von Lungenkarzinom-Patienten, die von einer zielgerichteten Therapie einen erheblichen Nutzen haben.

\section{Pathologie \\ $\nabla$}

Auf Basis groß angelegter molekularer Screeningstudien wurden unterschiedliche Faktoren wie z.B. die anaplastische Lymphomkinase (ALK) 

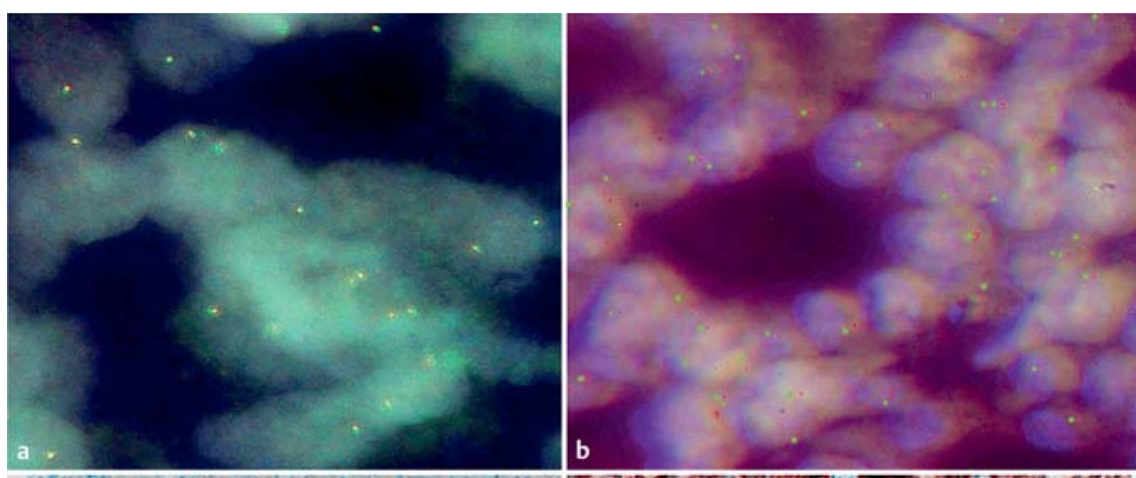

Abb. 1 Beispiele von Fluoreszenz-in-situ-Hybridisierung (FISH) und Immunhistochemie.

a FISH mit fusionierten roten und grünen Signalpaaren (gelb), kein Nachweis einer ALK-Translokation; b FISH, Nachweis einer ALK-Translokation. Die Trennung der roten und grünen Signale ist deutlich zu erkennen; c immunhistochemisch schwache Reaktion, geringe ALK-Expression; d immunhistochemisch starke Reaktion, deutliche ALK-Expression.
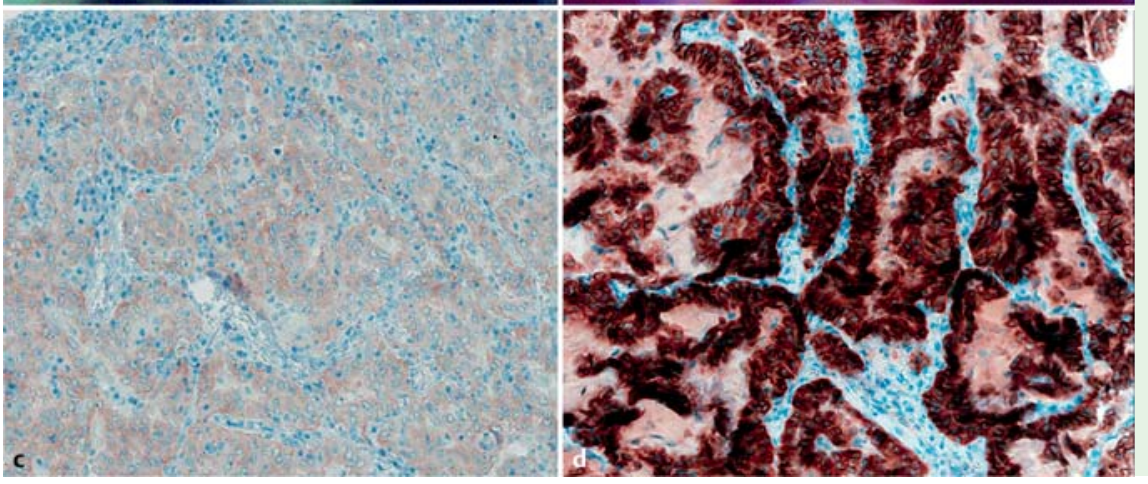

identifiziert, welche ggf. eine zielgerichtete Tumortherapie des pulmonalen nicht-kleinzelligen Karzinoms (NSCLC) ermöglichen [1]. Entsprechende Alterationen von ALK finden sich in 3\% [2,3] der Adenokarzinome (ADC), vorwiegend bei jungen Nichtrauchern (never/light smokers) mit EGFR- und K-RAS-Wildtyp [4,5], teilweise jedoch auch in Plattenepithelkarzinomen (SQCC) [1,6] und adeno-squamösen Karzinomen (ASC) [7]. Auf Basis der Literaturdaten und unserer eigenen Beobachtungen kommen ALKTranslokationen im Wesentlichen in prädominant azinären oder prädominant soliden Adenokarzinomen vor [1,8-11], wobei auch eine Assoziation zu anderen Tumordifferenzierungen beschrieben ist [3].

Die ALK-Expression ist assoziiert mit einem genomischen Rearrangement (Inversion), in dessen Folge die Kinasedomäne von ALK unter die Promotorkontrolle von EML4 (echinoderm microtubule-associated protein-like 4 ) gerät $[1,5]$. Der chromosomale Bruchpunkt innerhalb von EML4 tritt in unterschiedlichen Bereichen auf, was zu zahlreiche Varianten von EML4-ALK-Fusionen führt [12]. In seltenen Fällen variiert auch der Bruchpunkt innerhalb von ALK [13]. Weitere zwischenzeitlich identifizierte Fusionspartner von ALK sind u.a. TFG [14] und KIF5B [15, 16]. Neben der Translokation von ALK sind Amplifikationen sowohl von ALK als auch von EML4 beschrieben [2, 17], wobei eine alleinige ALKAmplifikation offenbar nicht zwingend zu einer Expression von ALK auf Proteinebene führt [17]. Jüngere Untersuchungen zeigen zudem, dass in NSCLC-Tumorzellen neben einer aktivierenden EML4-Translokation auch weitere molekulare Veränderungen detektiert werden können, wie eine aktivierende EGFR-Mutation, ALK-Amplifikation oder Mutationen in der ALK-Kinase-Domäne [18]. Viele dieser Veränderungen gelten als möglicher Resistenzmechanismus gegenüber einer Therapie mit Crizotinib, allerdings bleiben der klinische Stellenwert und die Prävalenz dieser weiteren molekularen Veränderungen aktuell noch unklar.

\section{Diagnostik der ALK-Translokation \\ $\nabla$}

Erste klinische Studien zeigen eine signifikante Tumormassenreduktion in den meisten NSCLC-Patienten mit ALK-Translokation, welche mit entsprechenden Inhibitoren behandelt wurden [19]. Der ALK-Status dieser Patienten wurde mittels Fluoreszenz-in-situ-Hybridisierung (FISH) bestimmt, wobei hierbei der ALK-Genlokus von zwei fluoreszenzmarkierten Sonden (z.B. grün und rot) flankiert ist. Durch die Inversion vergrößert sich der Abstand zwischen den Sonden, wobei hier ein Abstand von 2 Sondendurchmessern zwischen den beiden Signalen als diagnostisches Kriterium für eine Translokation gilt ( $\bullet$ Abb. 1). FISH ist demnach aktuell der Goldstandard, um Patienten zu selektieren, die möglicherweise von ALK-Inhibitoren profitieren $[4,19]$. Bei alleiniger FISH-basierter Diagnostik kann jedoch problematisch sein, dass der Abstand des invertierten ALK-Genlokus sehr kurz ist ( 12 Megabasen) und nur etwa 5\% der Gesamtlänge von Chromosom 2 betrifft. Da die Inversion ein dreidimensionales Ereignis ist, in der FISH aber nur zweidimensional dargestellt werden kann, kann die Interpretation des Sondenabstandes, insbesondere bei nur wenig vorhandenen Tumorzellen, deutlich erschwert sein. FISH kann daher potenziell zu falsch negativen Ergebnissen führen [9], weshalb ein kombinierter diagnostischer Ansatz mit weiteren Methoden sinnvoll erscheint $[9,20]$. Da ALK-Inhibitoren gegen ALK-Proteine und nicht spezifisch gegen eine ALK-Translokation gerichtet sind, könnte perspektivisch der Nachweis einer ALK-Expression auf Proteinebene genügen, um Patienten mit ALK-Inhibitoren zu behandeln. Potenziell könnten z.B. auch epigenetische Veränderungen in Tumoren zu einer ALK-Expression führen. Das Ansprechen entsprechender Tumoren auf ALK-Inhibitoren ist Gegenstand aktueller Studien.

Als ergänzende Methoden der ALK-Diagnostik bieten sich neben der Immunhistochemie auch PCR-basierte Ansätze mit spezifischem Nachweis entsprechender Fusionstranskripte an, z.B. auf Basis einer Multiplex RT-PCR [21] oder einem Exon-Array-Profiling [12,22] ( Tab.1). Bei diesen Ansätzen besteht jedoch die Problematik, dass in der Regel nur bekannte EML4-ALK-Varian- 
Tab. 1 Vergleich verschiedener Labormethoden zum Nachweis einer EML4-ALK Translokation.

\begin{tabular}{|c|c|c|c|}
\hline & Immunhistochemie & FISH & RT-PCR \\
\hline Vorteile & $\begin{array}{l}\text { - kostengünstig } \\
\text { - einfach auszuwerten } \\
\text { - überall verfügbar }\end{array}$ & $\begin{array}{l}\text { - Goldstandard in bisherigen Studien } \\
\text { - gute Sonden verfügbar (FDA approved) }\end{array}$ & $\begin{array}{l}\text { - } \text { kostengünstig } \\
\text { - spezifischer Nachweis ggf. auch bei } \\
\text { wenig Tumormaterial möglich }\end{array}$ \\
\hline Nachteile & $\begin{array}{l}\text { - geringe Sensitivität einiger Antikör- } \\
\text { per, ggf. in einzelnen Fällen negativ, } \\
\text { weitere Validierung erforderlich } \\
\text { - kein etabliertes Scoring-System } \\
\text { - Ansprechen positiver Fälle auf } \\
\text { ALK-Inhibitoren nicht abschließend } \\
\text { geklärt }\end{array}$ & $\begin{array}{l}\text { - teuer } \\
\text { - Interpretation schwierig } \\
\text { - nicht überall verfügbar }\end{array}$ & $\begin{array}{l}\text { - ggf. nicht überall verfügbar } \\
\text { - technisch anspruchsvoll } \\
\text { - nur bekannte EML4-ALK-Varianten und } \\
\text { Fusionen mit weiteren Translokations- } \\
\text { partnern können nachgewiesen werden } \\
\text { - keine Studiendaten verfügbar } \\
\text { (Ansprechen auf ALK-Inhibitoren) }\end{array}$ \\
\hline
\end{tabular}

ten bzw. ALK-Translokation mit anderen Fusionspartnern nachgewiesen werden können. Alleine die bis heute berichtete Anzahl unterschiedlicher Varianten und Fusionspartner spricht somit eher gegen diese Methodik. Die Immunhistochemie ist eine verlässlich durchführbare und kostengünstige Methode der gewebsbasierten Diagnostik, welche in Deutschland flächendeckend zur Verfügung steht. Ein Ringversuch zur Detektion ALK-positiver NSCLC wurde aktuell von der Qualitätssicherungs-Initiative Pathologie durchgeführt (http://www.quip-ringversuche.de) und abgeschlossen. 36 Institute erhielten ein Qualitätszertifikat. Initial bestand das Problem, dass die zur Verfügung stehenden ALK-Antikörper für die Lymphom-Diagnostik etabliert wurden, die ALK-Expressionslevel im NSCLC jedoch deutlich geringer sind. Zwischenzeitlich wurde jedoch ein offenbar sensitiverer Antikörper entwickelt [23], welcher nun auch kommerziell erhältlich ist. Prinzipiell besteht auch nach unseren eigenen Erfahrungen eine hohe Konkordanz zwischen ALK-Translokationen und der immunhistochemisch nachweisbaren ALK-Expression $[3,6,19]$.

\section{Klinik}

$\nabla$

Patienten mit EML4-ALK-Translokation sind meist jünger und Nichtraucher. Selten besteht eine weitere Mutation wie EGF-R oder KRAS.

Die Halbwertszeit von Crizotinib beträgt ca. 42 h, und die Resorption ist relativ unabhängig von den Mahlzeiten. Bei einer Dosis von $250 \mathrm{mg}$ zweimal täglich ist ein „steady state“ im Plasma nach 15 Tagen erreicht. Die Substanz wird in der Leber über CYP3A4 metabolisiert und ist ein moderater CYP-Inhibitor. Daher die Empfehlung, starke CYP-Induktoren (z. B. Carbamazepin, Phenytoin) und Inhibitoren (z. B. Makrolide, Azole, Grapefruitsaft) zu meiden. Darüber hinaus wurde eine leichte Bradykardie unter Crizotinib ohne QTc-Verlängerung beobachtet, sodass ein EKG unter Substanzen wie Ciprofloxacin empfohlen wurde.

$\mathrm{Zu}$ den häufigsten möglichen unerwünschten Wirkungen gehören Sehstörungen (62\%), Übelkeit (53\%), Durchfall (43\%), Erbrechen (40\%), Ödeme (28\%), Verstopfung (27\%) und Müdigkeit (20\%). Über eine Veränderung der Leberwerte, eine Verlängerung des QT-Intervalls und vereinzelt eine lebensbedrohliche Pneumonitis wurde berichtet.

\section{Studien \\ $\nabla$}

Präklinische Studien wiesen Crizotinib als potenten ALK-Inhibitor aus. Initial zeigte sich jedoch eine Aktivität als c-MET-Inhibitor, der HGF-vermitteltes Endothelzellüberleben, Apoptose und Angiogenese blockierte. Eine stärkere Antitumoraktivität fand sich allerdings bei Zelllinien mit EML4-ALK-Translokation, wobei Zelllinien ohne ALK-Translokation kein Ansprechen auf Crizotinib zeigten.

Ein Reihe von zum Teil noch laufenden klinischen Studien haben vielversprechende Daten zu Crizotinib geliefert [24], sodass die Zulassung im November 2012 auf Grund dieser Ergebnisse durch die EMA in Europa erfolgte.

Die Phase-I-Studie [25] (Profile 1001) untersucht bei ca. 400 Patienten mit ALK-Translokation die Dosiseskalation bei Patienten mit soliden Tumoren. Zuletzt wurden NSCLC-Patienten eingeschlossen mit einer Dosis von $250 \mathrm{mg}$ zweimal täglich. In der Phase-II-Studie (Profile 1005) wurden ebenfalls über 400 Patienten mit vorbehandeltem NSCLC untersucht, die ALK-FISH-positiv waren. Hier wurde ebenfalls eine Dosis von $250 \mathrm{mg}$ zweimal täglich verwendet. Beide Studien zeigten eine Ansprechrate von 60 bzw. 53\%. Das progressionsfreie Überleben betrug 9,2 beziehungsweise 8,5 Monate.

Die beiden Phase-III-Studien untersuchen Crizotinib versus Pemetrexed bzw. Docetaxel bei vorbehandelten Patienten (Profile 1007) bzw. in der ersten Linie versus Platin/Pemetrexed (Profile 1014). In beiden Studien wurden über 300 Patienten eingeschlossen. Erste Ergebnisse wurden beim ESMO 2012 präsentiert und zeigen einen Vorteil für Crizotinib mit einer Verlängerung des progressionsfreien Überlebens auf 7,7 Monate im Vergleich zu 3,0 Monaten im Pemetrexed- bzw. Docetaxel-Arm (HR 0.49; $\mathrm{p}<0.0001)$. Die Ansprechrate war ebenfalls höher mit Crizotinib (65\% vs 20\%; p<0.0001).

\section{Therapieversagen \\ $\nabla$}

Bei einem durchschnittlichen Ansprechen auf Crizotinib von ca. 8-9 Monaten stellt sich die Frage, warum dieser Effekt nicht länger aufrechtzuerhalten ist. Ähnlich wie bei der EGF-R-Mutation scheint es hier genetische Veränderungen zu geben, die einen Resistenzmechanismus vermitteln und über die Mutation von C1156Y und L1196M in ALK zu einer reduzierten Wirksamkeit von Crizotinib führen [18,26-29]. Möglicherweise werden ALKInhibitoren der nächsten Generation, die jetzt in Entwicklung sind, hier eine höhere Aktivität haben. Darüber hinaus gibt es Daten zur Veränderung des Tumorgenoms bei Patienten, die 
unter Crizotinib progredient werden und neue Mutationen, wie z.B. EGF-R-Mutation, erwerben bzw. die ALK-Translokation verlieren. Somit kann also bei Progress unter Crizotinib im Rahmen von Studien eine erneute Biopsie mit Evaluation der Mutationen erforderlich sein, um weitere Therapieoptionen zu erhalten.

\section{Fazit}

Zusammenfassend ist der ALK/c-MET-Inhibitor Crizotinib bei vorbehandelten NSCLC-Patienten mit einer Translokation von EML4-ALK der zweite zugelassene zielgerichtete molekulare Therapieansatz beim fortgeschrittenen NSCLC. Eine enge Kooperation mit dem Pathologen ist erforderlich, um eine qualitativ hochwertige und schnelle Diagnostik zu ermöglichen.

\section{Interessenkonflikt}

Die Autoren geben an, dass kein Interessenkonflikt besteht.

\section{Literatur}

1 Soda $M$, Choi $Y L$, Enomoto $M$ et al. Identification of the transforming EML4-ALK fusion gene in non-small-cell lung cancer. Nature 2007; 448: $561-566$

2 Perner S, Wagner PL, Demichelis F et al. EML4-ALK fusion lung cancer: a rare acquired event. Neoplasia 2008; 10: 298 - 302

3 Inamura K, Takeuchi K, Togashi Yet al. EML4-ALK fusion is linked to histological characteristics in a subset of lung cancers. J Thorac Oncol 2008; $3: 13-17$

4 Shaw AT, Yeap BY, Mino-Kenudson $M$ et al. Clinical features and outcome of patients with non-small-cell lung cancer who harbor EML4ALK. J Clin Oncol 2009; 27: 4247-4253

5 Sasaki T, Rodig SJ, Chirieac LR et al. The biology and treatment of EML4ALK non-small cell lung cancer. Eur J Cancer 2010; 46: 1773 - 1780

6 Boland JM, Erdogan S, Vasmatzis G et al. Anaplastic lymphoma kinase immunoreactivity correlates with ALK gene rearrangement and transcriptional up-regulation in non-small cell lung carcinomas. Hum Pathol 2009; 40: $1152-1158$

7 Wong DW, Leung EL, So KK et al. The EML4-ALK fusion gene is involved in various histologic types of lung cancers from nonsmokers with wild-type EGFR and KRAS. Cancer 2009; 115: 1723-1733

8 Inamura K, Takeuchi K, Togashi Yet al. EML4-ALK lung cancers are characterized by rare other mutations, a TTF- 1 cell lineage, an acinar histology, and young onset. Mod Pathol 2009; 22: 508-515

9 Rodig SJ, Mino-Kenudson M, Dacic $S$ et al. Unique clinicopathologic features characterize ALK-rearranged lung adenocarcinoma in the western population. Clin Cancer Res 2009; 15: 5216-5223

10 Jokoji R, Yamasaki T, Minami S et al. Combination of morphological feature analysis and immunohistochemistry is useful for screening of EML4-ALK-positive lung adenocarcinoma. J Clin Pathol 2010; 63: $1066-1070$
11 Koivunen JP, Mermel C, Zejnullahu K et al. EML4-ALK fusion gene and efficacy of an ALK kinase inhibitor in lung cancer. Clin Cancer Res 2008; 14: $4275-4283$

12 Sanders HR, Li HR, Bruey JM et al. Exon scanning by reverse transcriptase-polymerase chain reaction for detection of known and novel EML4-ALK fusion variants in non-small cell lung cancer. Cancer Genet 2011; 204: $45-52$

13 Penzel R, Schirmacher P, Warth A. A novel EML4-ALK variant: exon 6 of EML4 fused to exon 19 of ALK. J Thorac Oncol 2012; 7: 1198-1199

14 Rikova K, Guo A, Zeng Q et al. Global survey of phosphotyrosine signaling identifies oncogenic kinases in lung cancer. Cell 2007; 131: 1190 1203

15 Takeuchi K, Choi YL, Togashi Yet al. KIF5B-ALK, a novel fusion oncokinase identified by an immunohistochemistry-based diagnostic system for ALK-positive lung cancer. Clin Cancer Res 2009; 15: 3143 - 3149

16 Wong DW, Leung EL, Wong SK et al. A novel KIF5B-ALK variant in nonsmall cell lung cancer. Cancer 2011; 117: 2709-2718

17 Salido M, Pijuan L, Martinez-Aviles L et al. Increased ALK gene copy number and amplification are frequent in non-small cell lung cancer. J Thorac Oncol 2011; 6: 21-27

18 Doebele RC, Aisner DL, Le AT et al. Analysis of resistance mechanisms to ALK kinase inhibitors in ALK+ NSCLC patients. J Clin Oncol 2012; 30: 7504

19 Kwak EL, Bang YJ, Camidge DR et al. Anaplastic lymphoma kinase inhibition in non-small-cell lung cancer. N Engl J Med 2010; 363: 1693 1703

20 Yi ES, Boland JM, Maleszewski JJ et al. Correlation of IHC and FISH for ALK gene rearrangement in non-small cell lung carcinoma: IHC score algorithm for FISH. J Thorac Oncol 2011; 6: 459-465

21 Takeuchi K, Choi YL, Soda $M$ et al. Multiplex reverse transcription-PCR screening for EML4-ALK fusion transcripts. Clin Cancer Res 2008; 14: $6618-6624$

22 Lin E, Li L, Guan Yet al. Exon array profiling detects EML4-ALK fusion in breast, colorectal, and non-small cell lung cancers. Mol Cancer Res 2009; 7: $1466-1476$

23 Mino-Kenudson M, Chirieac LR, Law $K$ et al. A novel, highly sensitive antibody allows for the routine detection of ALK-rearranged lung adenocarcinomas by standard immunohistochemistry. Clin Cancer Res 2010; $16: 1561-1571$

24 Scagliotti G, Stahel RA, Rosell R et al. ALK translocation and crizotinib in non-small cell lung cancer: An evolving paradigm in oncology drug development. Eur J Cancer 2012; 48: 961 -973

25 Camidge DR, Bang Y-J, Kwak EL et al. Activity and safety of crizotinib in patients with ALK-positive non-small-cell lung cancer: updated results from a phase 1 study. Lancet Oncol 2012; 13: 1011 -1019

26 Zhang S, Wang F, Keats J et al. Crizotinib-resistant mutants of EML4ALK identified through an accelerated mutagenesis screen. Chem Biol Drug Des 2011; 78: 999-1005

27 Doebele RC, Pilling AB, Aisner D et al. Mechanisms of resistance to crizotinib in patients with ALK gene rearranged non-small cell lung cancer. Clin Cancer Res 2012; 18: 1472 - 1482

28 Katayama R, Shaw AT, Khan TM et al. Mechanisms of acquired crizotinib resistance in ALK-rearranged lung cancers. Sci Transl Med 2012; 4: 120ra17

29 Sasaki T, Koivunen J, Ogino A et al. A novel ALK secondary mutation and EGFR signaling cause resistance to ALK kinase inhibitors. Cancer Res 2011; 71: 6051-6060 